\title{
Influence of magneto-optic effects on light self-focusing in Kerr media
}

\author{
Katarzyna A. Rutkowska, ${ }^{* 1}$ Jerzy Jasiński, ${ }^{1}$ Krzysztof Świtkowski, ${ }^{1}$ Boris A. Malomed, ${ }^{2}$ and Roberto Morandotti ${ }^{3}$ \\ ${ }^{1}$ Faculty of Physics, Warsaw University of Technology, 00-662 Warsaw, POLAND, \\ ${ }^{2}$ Department of Physical Electronics, Faculty of Engineering, Tel Aviv University, Tel Aviv 69978, ISRAEL, \\ ${ }^{3}$ INRS-EMT, 1650 Blvd. Lionel Boulet, Varennes (Québec), J3X 1S2 CANADA.
}

Received March 20, 2012; accepted March 29, 2012; published March 31, 2012

\begin{abstract}
We present the possibility of light beam-propagation control in a Kerr nonlinear medium through the efficient management of magnetically-induced linear and circular birefringences. Theoretical analyses, performed by dint of direct numerical simulations of modified nonlinear Schrödinger equation, as well as variational approximation, show consistent results.
\end{abstract}

High-power optical beams in bulk self-focusing Kerr media are subject to instabilities that may lead to a catastrophic collapse [1-2]. It occurs when the input optical power exceeds the threshold (critical) value. In the past decade, the collapse of optical beams in the Kerr-type media has been intensively studied, mainly in the context of control and complete elimination of the collapse. To this end, different methods for collapse management have been proposed and theoretically implemented using suitable modifications of the nonlinear Schrödinger equation (NLSE) [3]. The modifications represent additional effects and phenomena in the respective physical model. The modified NLSE may result in the stable propagation of spatial solitons in bulk Kerr media.

In this work, we study theoretically a possible method for collapse management achieved by means of a suitable combination of linear $\left(\Delta n_{l}\right)$ and circular $\left(\Delta n_{c}\right)$ optical birefringences. Specifically, magneto-optical effects are taken as a particular physical example, which enables the introduction of both types of birefringences simultaneously via the superposition of the CottonMouton and the Faraday effects [4]. In this paper, using both numerical simulations for the modified NLSEs and the variational approximation (VA) applied to the same system, we show efficient management of the collapse, which in specific cases may result in the suppression of the collapse. Our analyses show that, for a fixed value of optical power (above the critical one), the proper application of the magnetic field may change the beampropagation dynamics. However, complete stabilization of bimodal beams propagating in the form of two-

*E-mail: kasia@if.pw.edu.pl dimensional solitary waves has not been obtained under the proposed conditions. Instead, quasi-stabile beams with their widths oscillating in the course of propagation (with the onset of the collapse postponed to more than tens of thousands of diffractions lengths) have been predicted (see e.g. Fig. 3 and Fig. 6).

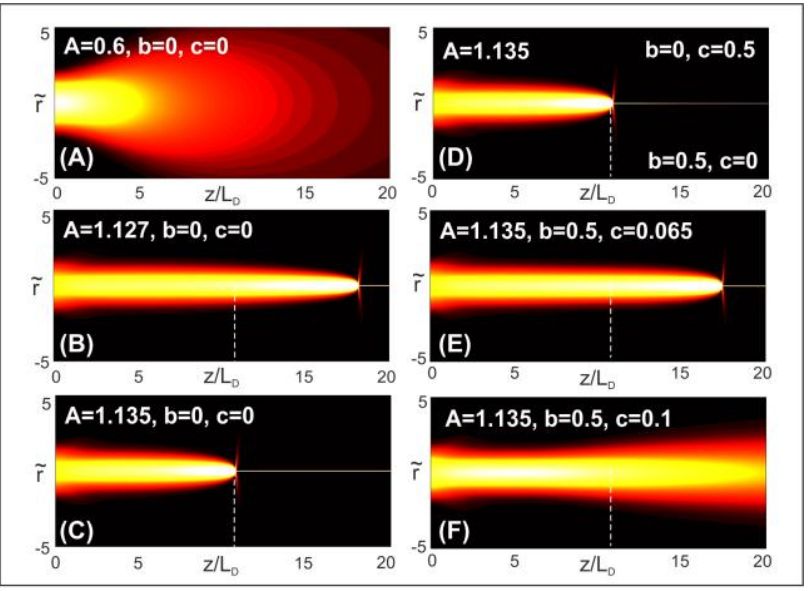

Fig. 1. Typical results of numerical simulations [based on Eqs. (1)], showing the dependence of beam propagation on input optical power (related to a normalized amplitude A of the initial Gaussian beam profile) and on the birefringence coefficients b, c. When the power increases (A-C), a spatial divergence of the beam may be reduced and eventually (when power exceeds the critical value) collapse of the beam is observed (with a distance for the onset of the collapse shorter for higher powers). For constant optical power, different variants of the beam-propagation dynamics occur, depending on the birefringence coefficients (C-F).

Our theoretical model is based on a system of coupled (normalized) scalar modified NLSEs, derived for the fundamental beams (i.e. those with zero vorticity) in the presence of the self-focusing Kerr nonlinearity, under the assumption of the circular symmetry:

$$
\begin{aligned}
i \frac{\partial u_{R, L}}{\partial \tilde{z}}+\frac{1}{2}\left(\frac{\partial^{2}}{\partial \tilde{r}^{2}}\right. & \left.+\frac{1}{\tilde{r}} \frac{\partial}{\partial \tilde{r}}\right) u_{R, L} \pm b u_{R, L}+c u_{L, R}+ \\
& +\left(\left|u_{R, L}\right|^{2}+2\left|u_{L, R}\right|^{2}\right) u_{R, L}=0,
\end{aligned}
$$


with typical normalization factors applied [1]. Here $u_{R, L}$ are the scaled complex slowly-varying envelopes of the right- and left- circularly polarized components, $\tilde{r}=r / w_{0}, \tilde{z}=z /\left(2 L_{D}\right)$ are the normalized transverse and longitudinal coordinates, $w_{0}$ is the initial width of the (Gaussian) beam, $L_{D}$ is the diffraction length. Terms with real coefficients $b$ and $c$ describe the magneticallyinduced circular and linear birefringences, respectively (i.e. $b \sim \Delta n_{c}$ and $c \sim \Delta n_{l}$ ). Since the propagation equations (Eqs. 1) support collapsing solutions, which can be predicted for certain geometry and initial conditions, detailed numerical simulations were performed in order to determine how the additional terms (including the birefringence coefficients) in NLSE may stabilize or destabilize the beam against the collapse. Typical results of numerical simulations are summarized in Figs. 1-2. As one can see, when both coefficients are present in a proper ratio (i.e. for a particular combination of the magneticallyinduced birefringences), the collapse is suppressed, or the distance which the beam passes before the onset of the collapse may be efficiently extended (see Figs. 1E-F, 2C) with respect to the case of the same optical power associated with the input beam, but without the external magnetic field applied (Fig. 1C). The effect of the combined birefringences for a high-intensity beam with a fixed normalized amplitude $(A=1.135)$ is schematically shown in Figs. 2A-B, demonstrating the tunability of the collapse in the $(b, c)$-plane. Note that the black color in Figs. 2A-B (and then also in Fig. 3A) indicates the output widths that are close to the initial value $w / w_{0} \approx 1$, defining thus the range of the birefringence coefficients for which the propagating beam could be potentially stable. As shown in Figs. 2-3, at a given value of the input power, the delay and/or the suppression of the collapse may be expected at the collapse-diffraction (for $c>b$ ) and diffraction-collapse (for $b>c$ ) borders.

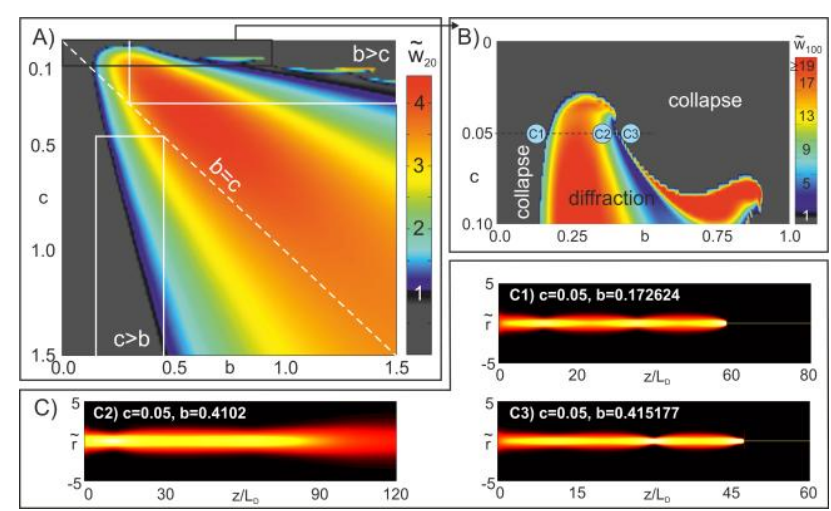

Fig. 2. The normalized width of the output beam (for an input amplitude $\mathrm{A}=1.135)$ and the propagation distance $z_{M A X}=20 L_{D}(\mathrm{~A})$ and $z_{M A X}=100 L_{D}$ (B), as a function of the birefringence coefficients. Solutions that collapse at $\mathrm{z}<z_{M A X}$ are represented by the gray color in the corresponding panels. Panel $\mathrm{C}$ shows the beam propagation for particular combinations of the birefringence coefficients as indicated in panel B.
In addition to numerical solutions based on the coupled NLSEs, the variational approximation [5] is also suitable for the description of beam propagation in bulk selffocusing Kerr media. The application of the VA significantly reduces the computation time, yet providing essential and accurate information about the collapse dynamics (as a function of the birefringence coefficients). In our case, the VA amounts to a system of three ordinary differential equations (ODEs) for the evolution of the normalized beam width, relative power and relative phase of the two polarization components (the underlying assumptions, detailed derivations and the final form of the mentioned equations can be found in Ref. [6]).

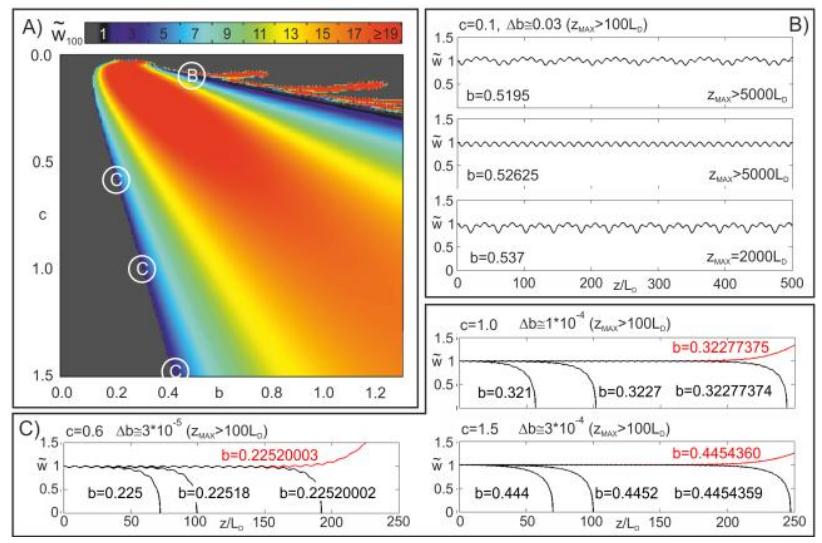

Fig. 3. Results of simulations performed in the framework of the VA. Initial normalized total optical power [6] has been taken as

$\tilde{N}=A^{2} \tilde{w}^{2} / 2=0.685$ for the best correspondence to the case presented in Fig. 2. As it was in the case of the direct numerical simulations of

NLSEs, quasi-stable results are observed for the birefringence coefficients at the boundary between diffracting and collapsing solutions (marked by B and C). Parameter $\Delta b$ in panels B-C indicates a range of the variation of the circular birefringence coefficient (for given c) within which the onset of the collapse takes place for the distance larger than $100 L_{\mathrm{D}}$.

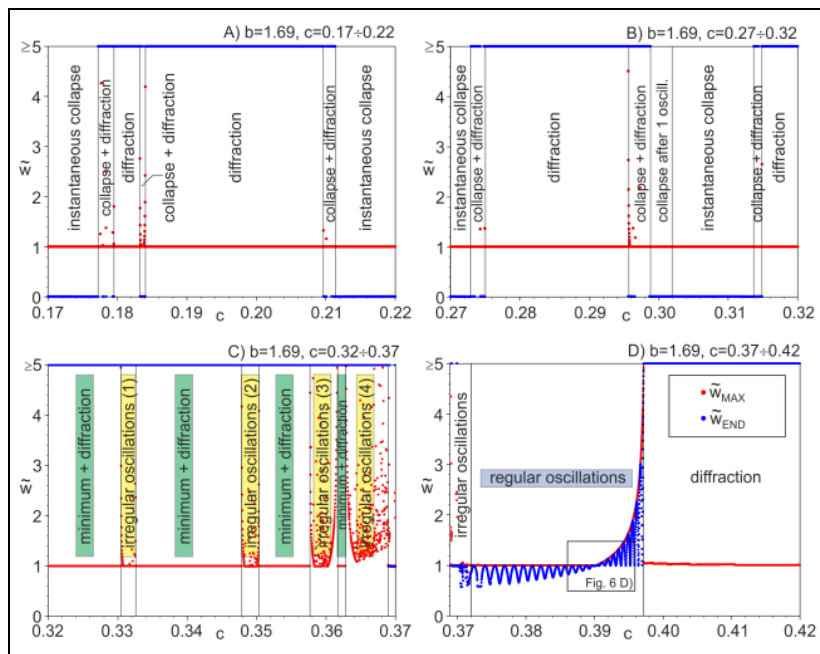

Fig. 4. Complicity of the solutions (obtained in the framework of the VA and representing changes of the beam width in the course of the propagation for $\tilde{\mathrm{N}}=0.685$ ) at the boundary between the collapse and 
diffraction (for the case when $\mathrm{b}>\mathrm{c}$ ). Here $b$ was set to 1.69 , for which slight changes in the value of the linear birefringence coefficient may lead to different beam-propagation dynamics as shown in Figs. 5-6 (where solutions in the form of instantaneous collapse or diffraction, similar to those in Fig. 1A-B, have been omitted). Conditions for the (quasi-) stabilization of the beam may be found for combinations of the birefringence coefficients for which regular oscillations of the beam width are observed (panel D) and the largest, the smallest and final (here obtained for $\mathrm{Z}_{\mathrm{MAX}}=5000 \mathrm{~L}_{\mathrm{D}}$ ) beam widths are the closest to the initial one (see also Fig. 6).
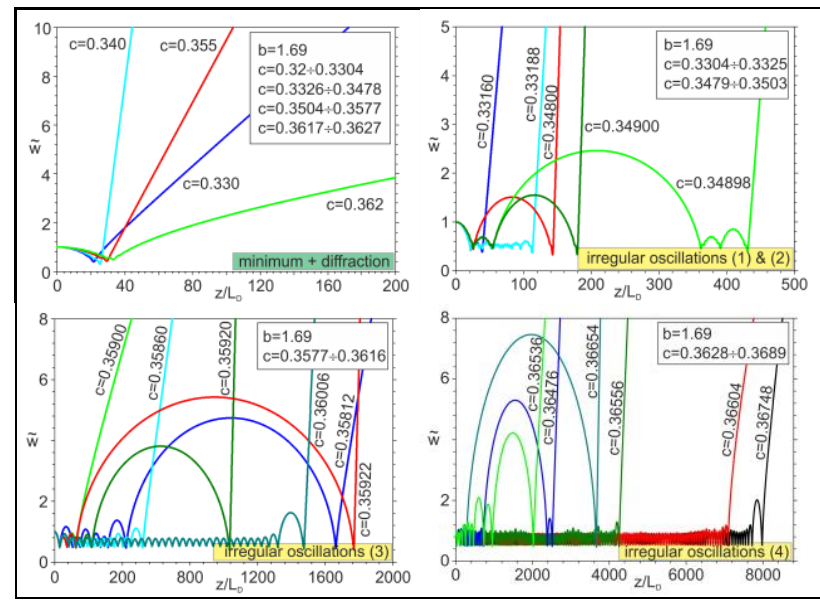

Fig. 5. Examples of different beam propagation dynamics obtained for fixed value of the circular birefringence coefficient $b$ and minor

changes of the linear birefringence coefficient $c$, showing the transition between diffracting and irregularly oscillating solutions. Further increment of $b$ allows regular oscillations of the beam width to be found (see Fig. 6).

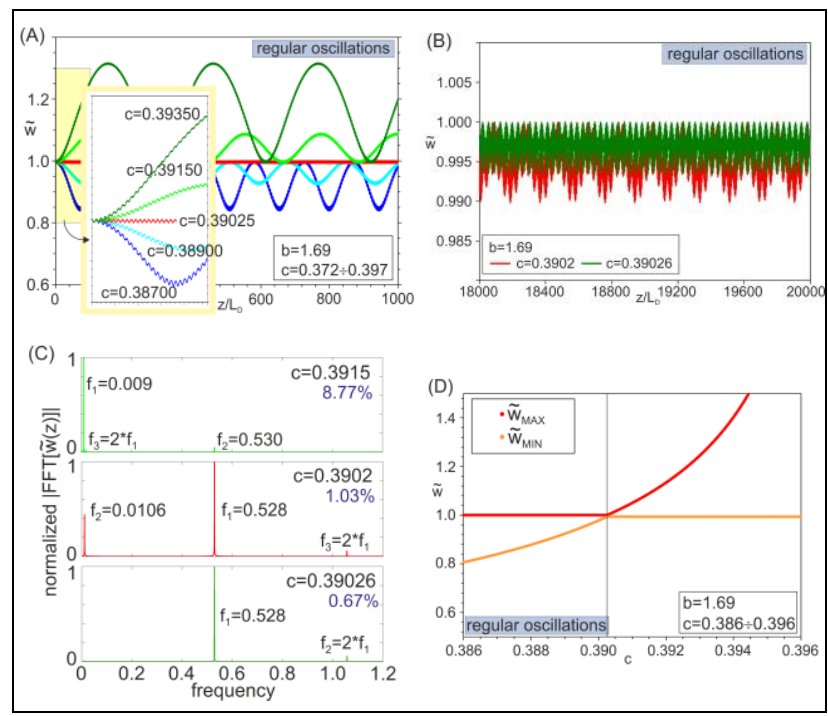

Fig. 6. Results for the regular oscillations of the beam width on the way of propagation. By precise matching the birefringence coefficients it is possible to find quasi-stable (oscillating) solutions for which beam width is slightly changing (see panels $\mathrm{B}, \mathrm{D}$ ). In panel C results of the Fourier transform performed on the beam width (as a function of the normalized propagation distance) are presented, showing the change of the frequency of the beam width oscillations [values written (in blue) in each graph (in $\mathrm{C}$ ) represents percentage changes of the beam width defined as: $\left.\left(\tilde{w}_{M A X}-\tilde{w}_{M I N}\right) / \tilde{w}_{M A X}\right]$

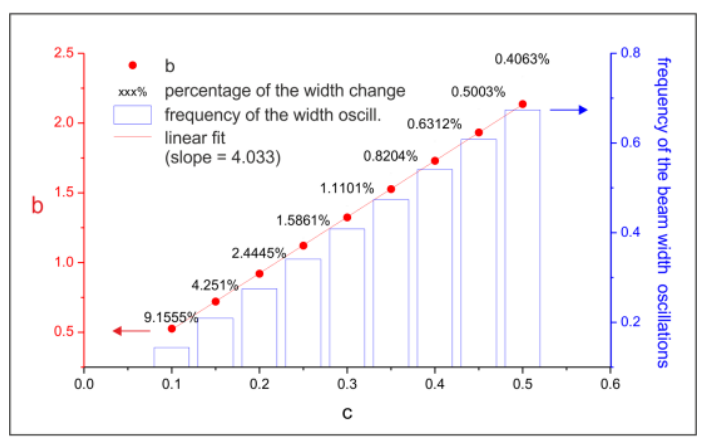

Fig. 7. Pairs of the birefringence coefficients $(c, b)$ corresponding to the quasi-stable oscillating solutions with the single period and the minimum amplitude of the oscillations of the beam width (red points). For the optical power considered (i.e. for $\tilde{\mathrm{N}}=0.685$ ) the estimated ratio is $b / c=4.033$. For each pair, the frequency of the beam width oscillations (bars) are given, together with percentage of the beam width changes (values over red points).

Detailed results of the theoretical analyses performed in the framework of the VA are presented in Figs. 3-7, showing a variety of possible solutions (depending on the precise adjustment of the birefringence coefficients) including quasi-stable ones [obtained for the specific ratio (constant for fixed optical power) of the birefringence coefficients].

In conclusion, we have shown theoretically that joint optical birefringences, as proposed here could be achieved in practice through a combined use of the Cotton-Mouton and Faraday effects, may effectively affect the dynamics of the optical collapse in bulk self-focusing media, leading to its postponement or arrest (for a fixed value of optical power). Our results offer new possibilities towards the use of magneto-optic effects for controlling various nonlinear phenomena. Moreover, because similar theoretical models are commonly used in other fields, such as e.g. sets of coupled Gross-Pitaevskii equations to describe BoseEinstein condensates, conclusions drawn from our theoretical analyses may be adopted to other physical media as well.

Katarzyna Rutkowska would like to acknowledge Iuventus Plus project (IP2010 036170) granted by the Polish Ministry of Science and Higher Education.

\section{References}

[1] R.W. Boyd, Nonlinear optics (San Diego, Academic Press 2008).

[2] C. Sulem, P.L. Sulem, The nonlinear Schrödinger equation: selffocusing and wave collapse (New York, Springer 1999).

[3] Y.S. Kivshar, D.E. Pelinovsky, Phys. Rep. 331, 117 (2000).

[4] R. Kurzynowski, W.A. Woźniak, Optik 115, 473 (2004).

[5] B.A. Malomed, Progr. Optics 43, 71 (2002).

[6] K.A. Rutkowska, B.A. Malomed, R. Morandotti, Opt. Express 18, 8879 (2010). 